\title{
Students' Visual Perceptions of Virtual Lectures as Measured by Eye Tracking
}

\author{
Yu-Jin Kim, Jin Ah Bae, and Byeong Ho Jeon \\ Dept. of Media Image Art \& Technology, Kongju National University, Sinkwandong, \\ Gongju, South Korea \\ \{yujinkim, jinabae, bhjeon\} @kongju.ac.kr
}

\begin{abstract}
In this paper, we used eye tracking methodologies to investigate students' visual perceptions of lectures using 3D real-time virtual studio technology. For measuring learning performance, we also gave the students multiple-choice paper quizzes at the end of the lectures. Three virtual lectures were created with different types of lecture materials (text-centered, image-centered, and lecturercentered) and 3D virtual sets (classroom, cyberspace, and lecture-theme space). Through analyzing students' eye movements in viewing still and moving scenes of the virtual lectures, we found that layouts and movements of design elements on lecture screens significantly influenced students' scanpaths and areas of interest (AOIs). Lecture material types affected learning performance while 3D virtual sets had no effect due to students' inattention to the virtual background areas. We discuss effective ways to develop virtual lectures and design lecture screens for better presentation of lecture content and higher learning performance.
\end{abstract}

Keywords: Virtual lectures, virtual studios, eye tracking, visual perception, learning performance, user-centered screen design.

\section{Introduction}

Over the last two decades, advances in digital technologies brought about the emergence of new teaching pedagogies such as Computer-Assisted Instruction (CAI), Computer-Supported Collaborative Learning (CSCL), Web-Based Instruction (WBI), and others. Besides these pedagogies using computer and internet technologies, there has been growing interest in virtual studio technology, which composites images of performers shot in front of a chroma-key blue background and visual images created through real-time 3D computer graphics [1]. The virtual studio technology increases face-to-face interaction between a lecturer and students and helps the lecturer interact with various types of lecture materials in dynamic virtual environments [2].

In academia, however, little attention has been paid to the methods to create courses using virtual studios for delivering learner-centered lecture materials and enhancing learning performance. To fill this research niche, this study aims to analyze how students look at the screens of virtual lectures [3] using eye tracking technology. Eye tracking provides insight into students' visual perceptions and cognitive processes by measuring their eye movements. We research how lecture material types and $3 \mathrm{D}$ virtual sets affect learning performance. 


\section{Related Work}

\subsection{Lectures Using Virtual Studio Technology}

Virtual education, by the use of cyberspace, eliminates the spatial and temporal limitations by removing the need for the lecturer and students to be present at an instructional site at a designated time [4]. Recently, the proliferation of information and communication technologies (ICTs) has spawned a boom in virtual education by creating a variety of virtual lecture content production techniques such as Flash, Web 3D, 3D real-time virtual studio, and others. Among these production technologies, several researchers have explored the effectiveness of the virtual studio application to lectures. In 2003, Morozov, Debelov, and Zhmulevskaya claimed that the application of virtual studio systems enhances the efficiency of instructional processes by providing lecturers more possibilities for diverse forms of education [5].

According to Brown and Cruickshank (2003), a virtual lecture can be more effective than classroom lecture by achieving significant savings in the costs of lecture delivery and student support, though a very large investment of time and effort is required by the lecturer [6].

Along with the previously mentioned research interests in the educational effects of the application of new technology, researchers have studied the factors affecting the delivery of lecture content in virtual education in order to increase the motivation and effects of learning. These factors include screen design [7, 8], content design [9], content suggestion style, and lecture material [10].

\subsection{Eye Tracking in Visual Information Processing}

Eye movements are driven both by properties of the visual world and processes in a person's mind [11]. Therefore, many scholars have used eye tracking techniques in order to explore the relations between eye movements and visual information processing in diverse research fields. In particular, researchers in HCI have tracked eye movements to understand visual and display-based information processing, as well as to discover the factors that may impact the usability of system interfaces [12]. In fact, eye movement data provide more detailed and specific information about a user's cognitive process in many different kinds of displays [13, 14]. They also help researchers determine the roots of some usability problems, and then come up with effective solutions.

Meanwhile, the recent trend of eye-tracking studies in HCI shows that eye-tracking methods have been rapidly employed in conducting usability testing of websites. Laarni et al. (2003) tracked the eye movements of users while they were selecting and reading online news items on a small computer [15]. Cowen (2004) asked the subjects to perform two tasks on a website and measured their total fixation duration, the number of total fixations, average fixation duration, and distribution of fixations on the screen [13]. Through these measurements, he suggested effective ways to design webpages in terms of user interface. In 2007, Cutrell and Guan also measured web surfers' eye movements to observe their web search strategies [16]. Like the aforementioned studies, many researchers have analyzed the interface of and interaction with web content, mainly focusing on a sequence of still scenes of web pages. 
However, there have been increasing interests in employing eye-tracking methods in moving visual images along with the proliferation of more dynamic web content, which is developed by adopting new types of web authoring tools and media, such as Flash, Web 3D, Virtual Studio, and others.

\subsection{Eye Tracking Metrics}

The main measurements used in eye-tracking research are "fixations," which are moments when eyes are relatively stationary, taking in or encoding information, and "saccades," which are quick eye movements occurring between fixations [12]. From these basic measurements, a multitude of metrics are also derived [17]: (1) "gaze duration," the cumulative duration and average spatial location of a series of consecutive fixations within an area of interest; (2) "area of interest (AOI)," the area of a display or visual environment that is of interest to the research or design team and, thus, defined by them (not by the participant); and (3) "scanpath," a special arrangement of a sequence of fixations.

\section{Hypotheses}

The focus of our study is the investigation of students' visual perceptions in virtual lectures. In order to identify the patterns of students' visual perceptions, we tracked their eye movements according to different layouts and movements of design elements on lecture screens. We also studied the effects of lecture material types and 3D virtual sets on students' learning performance. In line with these purposes, we investigated the following research hypotheses:

Hypotheses 1a. Students' eye movements (AOIs, scanpaths) are affected by the layouts of design elements on lecture screens.

Hypotheses 1b. Students' eye movements (AOIs, scanpaths) are affected by the movements of design elements on lecture screens.

Hypotheses 2a. Students' learning performance varies according to lecture material types.

Hypotheses 2b. Students' learning performance is different according to 3D virtual set types.

\section{Experiment}

\subsection{Virtual Lecture Prototypes}

In order to test the aforementioned hypotheses, we designed and conducted an experiment to analyze students' eye movements and learning performance in the context of virtual studio-based lectures. As experimental material, three virtual lecture prototypes were created under the theme of "Media and Culture" with varying layouts and movements of lecture screen design elements (lecturer, lecture board, 3D virtual sets, text, images, and movies on the lecture board). The three prototypes (see Figure 1) were also produced with different types of lecture materials (text-centered, image-centered, 
and lecturer-centered) and 3D virtual sets (classroom, lecture-theme space, and cyberspace): (1) Prototype 1 - text-centered lecture materials in classroom background sets; (2) Prototype 2 - lecturer-centered lecture materials in cyberspace background sets; and (3) Prototype 3 - image-centered lecture materials in lecture-theme space background sets. Figure 1 shows screen shots of these three prototypes. Three screen shots located in the first row display their 3D background sets. The other three shots in the second row illustrate how the same lecture content about the phenomena of mass communication is delivered by different types of lecture materials.
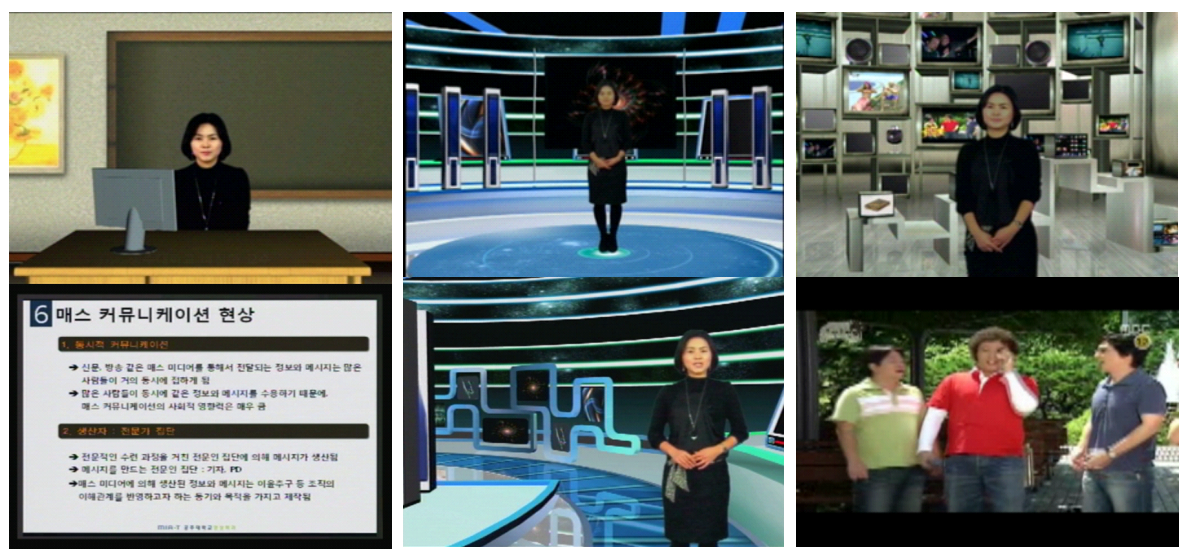

Fig. 1. Screen shots of three prototypes (left: Prototype 1, middle: Prototype 2, and right: Prototype 3)

After modeling and animating the above 3D virtual sets using 3D Studio Max 9.0., we combined these 3D background sources with the live-action footage of lecturers by a real-time chroma key technique of the "VS2000" system. In addition, we created animation buttons for controlling lecture screen design elements using the VS scripts of the "HotAction" program.

\subsection{Participants}

Forty participants were selected for the experiment, but ten of them had problem with the calibration of the eye-tracking system. Calibration was a fine-tuning process for the experiment, so 30 participants successfully completed the experiment. The 30 subjects were divided into three groups of ten who participated in the experiment composed of three kinds of virtual lectures. The subjects were freshmen and sophomores of K University and had almost no previous knowledge of the content of the experiment lectures. They were between the ages of 18 and 23, and the gender ratio was 1:1. Twenty-four of the students had experienced distance lectures, and eight of them had been exposed to VR lectures.

\subsection{Apparatus}

This experiment used the hardware "Eyegaze Development System" developed by LC Technologies, Inc. This device tracks the $x-y$ coordinates of the participant's 
gazepoint on the computer screen automatically using the pupil-center-corneal reflection (PCCR) method, which directs infrared light into the eye. This system generates raw eyegaze point location data at a camera field rate of $60 \mathrm{~Hz}[18]$ and calculates three kinds of gazepoints: (1) a moving point that a participant looks at for only $1 / 60$ second, then looking at other points; (2) a fixating point that a participant looks around in; and (3) a fixation completed point that a participant maintains the gaze in.

This study used the software "EyeTrack v1.0" and "EyeTrackMovie v1.0" for analyzing eye movements in viewing still and moving scenes, respectively. These programs were developed by the Human Computer Interaction Laboratory (HCIL) of KAIST for the visual monitoring and analysis of eye-tracking results that were expressed in coordinates [19]. "EyeTrack" and "EyeTrackMovie" provide "Replay" and "Analysis." "Fixation Mark" and "Color Variation" options enable the monitoring of eye-tracking data with a variety of graphic effects in chronological order.

For an efficient analysis of eye movement patterns, it also provides five analysis options: (1) "shadow," which shows the area that subjects focused on and illustrates the rest in shades with gradation effects; (2) "frequent area," which marks a clear boundary between the area that the subjects focused on and the rest, highlighting the former; (3) "hotspot," which shows the amount of gaze (red meaning more gaze and green meaning less gaze); (4) "selected area," which shows the amount of gaze in a certain area and its duration in numbers; and (4) "priority order," which shows the duration of a gaze over time in circles. All participants' eye movements could be monitored at once due to the function of the programs which can analyze multiple data simultaneously. The patterns of AOIs and scanpaths, described in Section 2.3, were discovered from these eye movements.

\subsection{Experimental Design and Procedure}

The experiment was performed in four stages, and it took about an hour for a participant to complete the experiment: (1) questionnaires on demographics, media education level, and cyber learning experiences; (2) still scene eye-tracking with the captured scenes according to distinguishable types of screen layouts (Prototype 1: 6 scenes, Prototype 2: 9 scenes; and Prototype 3: 7 scenes); (3) moving scene eyetracking with three prototype movies; and (4) a quiz with multiple-choice questions. Eye movements were tracked twice for still and moving scenes of virtual lectures. Compared with the eye-tracking of moving scenes, the eye-tracking of still scenes allowed more detailed and accurate analysis of eye movements for the different screen layout types. On the other hand, the eye-tracking of the moving scenes enabled an additional analysis of the effects of the movements of screen layout elements on eye movements.

\section{Results}

\subsection{AOIs According to the Layouts and Movements of Lecture Screen Design Elements}

Through analyzing students' eye movements in viewing still and moving scenes of the virtual lectures, we found that layouts and the movement of lecture screen design 
elements (the lecturer, lecture board, and 3D background set) significantly influenced students' scanpaths and areas of interest (AOIs) [H1a and H1b]. In the case of AOIs, parts that students paid close attention to were similar in both still and moving scene tests. By pointing out the design elements that received the most attention on the sill scenes, it was found that students generally gazed into a lecturer's face in all of the still scenes that feature a lecturer (Prototype 1: 4 scenes, Prototype 2: 5 scenes, and Prototype 3: 5 scenes) regardless of the lecturer's size and position on the screen. Figure 2 shows the "hotspot" option analysis results of the three scenes (left: Prototype 3, middle: Prototype 2, and right: Prototype 3), where different sizes of a lecturer appear in different positions. We calculated the distribution of fixations on the face of the lecturer in the left scene of Figure 2 using the "selected area" option and found that $52.5 \%$ of the total fixations were on the lecturer's face.

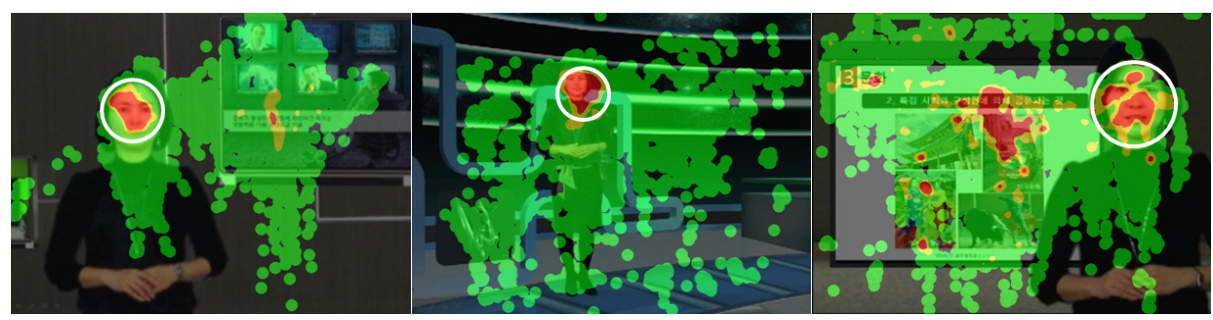

Fig. 2. "Hotspot" option analysis results (Note: The amounts of fixations gradually increased from green to red, and the reddest parts are marked in circle)

In addition, we found that the students' points-of-regard are likely to stay not just on the lecture's face, but also on the face of people illustrated in the lecture material images (see Figure 3). Another finding was that the student's attention to the people whose profile or back views were shown to decrease compared with front view images.
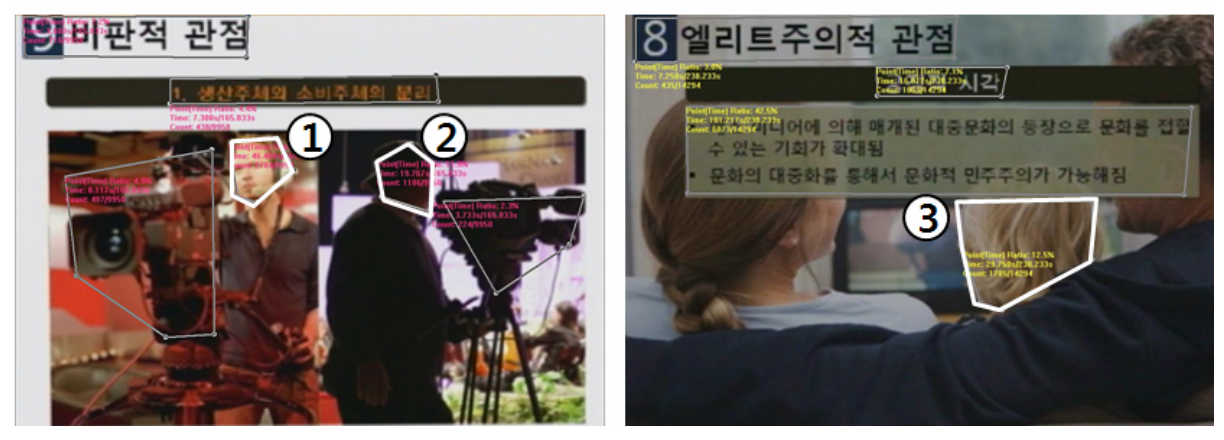

Fig. 3. "Selected area" option analysis results (1. Front view (28\%), 2. Profile view (11.9\%), and 3. Back view (12.5\%) of people in the image) 


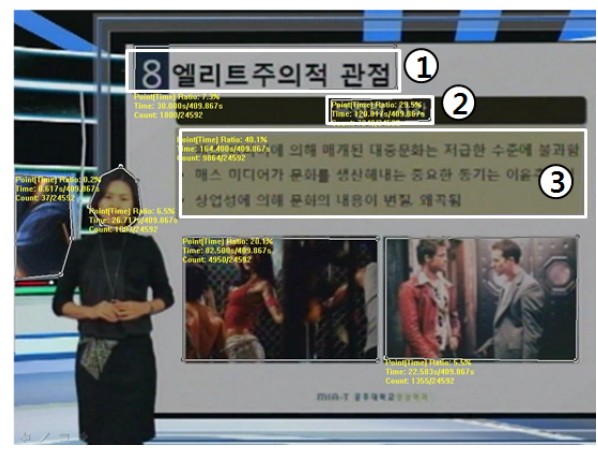

Fig. 4. Comparing visual attention to text areas $(3$. Body $(40.1 \%)>2$. Subtitle $(29.5 \%)>1$. Title $(7.3 \%))$

Along with the aforementioned visual attention to people's faces in the still scenes, we also found a tendency in the moving scenes that students' points-of regard are mostly directed at the lecturer's face as well as the faces of people illustrated in the lecture material images.

In addition, the analysis results also suggest that students' eye movements and text sizes in lecture boards were inversely proportional. There were less eye movements and shorter gazes on larger texts. In fact, visual attention was generally highest in the body, subtitles, and title (see Figure 4). In other words, considering that titles take up more space than subtitles, but receive less visual attention, it would be effective to deliver new and important messages using subtitles.

\subsection{Scanpaths According to the Layouts and Movements of Lecture Screen Design Elements}

In order to measure the student's scanpaths and scanpath duration, we selected a student whose eye movement pattern can represent the visual attention of the ten students participating in the same still scene experiment and then analyzed his/her eye movement over time using the "fixation map" and "priority order" options. From the results, it turned out that the farther from the center the objects are positioned, the
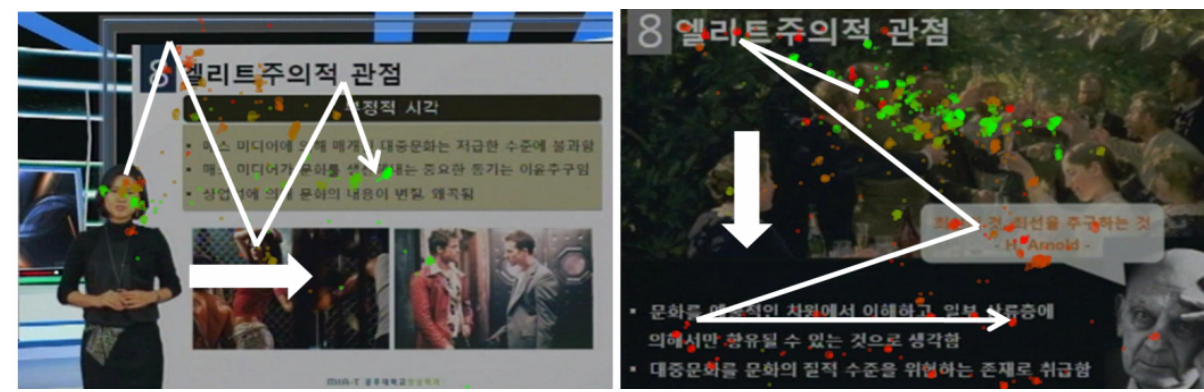

Fig. 5. Scanpath tracking (left: from left to right, right: from top to bottom) 
more likely they are to be out of the scanpath or stared at later. This analysis also verified the commonly accepted idea that people in Korean-speaking culture read words and sentences from left to right and from top to bottom (see Figure 5).

On the other hand, students' scanpaths showed different patterns in still and moving scene tests in terms of the starting point of the eye's gaze. In the still scene test, the starting points of the students' gazes were related to screen design element types, as well as their positions. For example, the majority of students firstly gazed into the lecturer's face, regardless of its position, and then moved their gazes to other elements located around the center of the screens. In the moving scene test, the movement of screen design elements, rather than their type, dominantly influenced the starting points, as illustrated in Figure 6. We also found that students moved their gazes to empty parts where the next lecture content was expected to show up. Properly timed anticipation could enable the students to better perceive animated lecture content by preparing them for what will come next in the lecture.

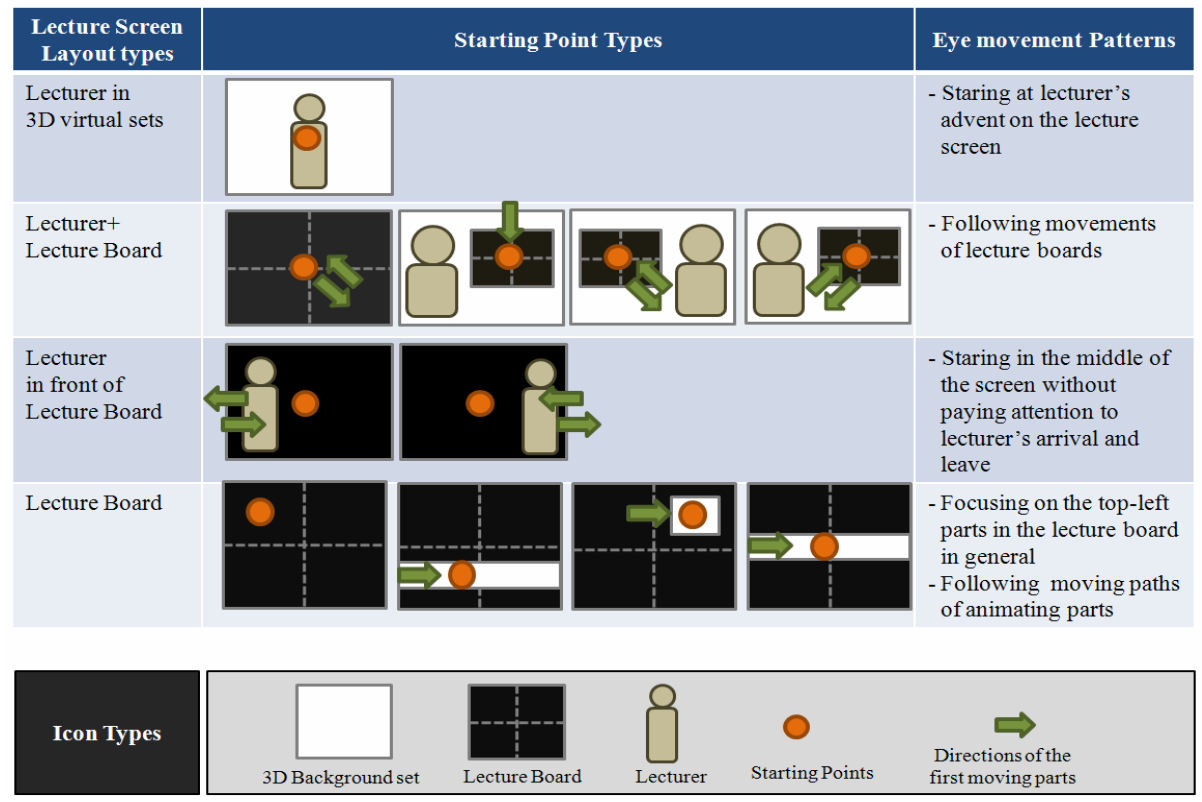

Fig. 6. Starting points of students' gazes according to the lecture screen layout type

\subsection{Students' Learning Performance According to Lecture Materials and 3D Virtual Sets}

The average quiz scores for the three groups who respectively watched text-centered, image-centered, and lecturer-centered virtual lectures $(12.9,9.7$, and 10.2, respectively) were significant at the alpha level of $0.05(\mathrm{~F}=4.694)$. These results support $\mathrm{H}$ $2 \mathrm{a}$, which suggests the possibility of a relationship between students' learning performance and lecture material types. Students could easily understand and memorize lecture content in text-centered lecture material which presented the lecturer's 
explanations in clear print on the screen. While lecture material types affected learning performance, 3D virtual sets had no effect on learning performance due to students' lack of attention to the background areas. Consequently, $\mathrm{H} 2 \mathrm{~b}$, which suggests a strong relationship between students' learning performance and 3D virtual set type, was not supported. In fact, the effects of the virtual sets on the learning process are very small because students' visual attention is usually drawn to the background only when they found some noticeable images or figures in the set.

\section{Conclusions}

This study investigated students' visual perceptions of virtual lectures by analyzing their AOIs and scanpaths in the still and moving scenes of the lectures. It also explored how to develop lecture materials and 3D background sets of virtual lectures for improving learning performance by testing the students' comprehension with multiple-choice questions. Our research revealed that the following issues on virtual lecture production should be carefully considered for better presentation of lecture content: (1) the lecturer's size and positions on lecture screens and people's postures illustrated in lecture materials; (2) text's sizes and positions in lecture materials; (3) movements of screen design elements (the lecturer, lecture board, animating parts on the lecture board); and (4) balanced layouts between the lecturer, lecture materials, and virtual background sets.

Educators and material designers should also consider that text-centered lecture materials were the most effective for higher learning performance than imagecentered and lecturer-centered ones. Even though statistical significance was not reached between 3D background sets and learning performance, an effective background set design suited to the lecture objectives and content is necessary for enhancing students' learning interest and motivation. Finally, it is hoped that this study will assist lecturers in understanding students' visual information processing in virtual lectures, in designing virtual lecture content more effectively, and in improving the instructional effects of virtual lectures.

\section{Acknowledgment}

We thank Kun-pyo Lee and Jung-mi Park for their comments and help in conducting our eye-tracking experiment.

\section{References}

1. Fukaya, T., Fujikake, H., Yamanouchi, Y., Mitsumine, H., Yagi, N., Inoue, S., Kikuchi, H.: An Effective Interaction Tool for Performance in the Virtual Studio-Invisible Light Projection System. NHK Science \& Technical Research Laboratories, Japan (2003)

2. Dolgovesov, B.S., Morozov, B.B., Shevtsov, M.Y.: The System for Interactive Virtual Teaching Based on "Focus" Virtual Studio. In: International Conference Graphicon 2003 in Moscow, Russia (2003) 
3. In this paper, a virtual lecture means instructional content that is created using virtual studio techniques for virtual education

4. Kuroda, K., Shanawez, H.D.: Strategies for Promoting Virtual Higher Education: General Considerations on Africa and Asia. Africa and Asian Studies 2(4), 565-575 (2003)

5. Morozov, B.S., Develov, B.B., Zhmulevskaya, M.Y.: The System for Interactive Virtual Teaching Based on "Focus" Virtual Studio. In: International Conference Graphicon, Moscow, Russia (2003), http: / / www. graphicon.ru /

6. Brown, S., Cruickshank, I.: The Virtual Studio. International Journal of Art \& Design Education 22(3), 281-288 (2003)

7. Kim, M.R.: Strategies on Screen Design of Learner-Centered Web-based Instructional Systems. Journal of Educational Technology 16(4), 51-65 (2008) (in Korean)

8. Shon, M., Chung, H.H.: An Analysis on the Learning Hindrance Factors in BlendedLearning Environment. Journal of Educational Information and Media 13(2), 251-276 (2007) (in Korean)

9. Ryu, I.: Factors Influencing the Effectiveness of Web-Based Distance Learning. Management Education Review 6(2), 7-27 (2003) (in Korean)

10. Kang, M.H., Gu, M.H., Moon, H.N., Jung, S.Y., Chung, J.Y., Kim, J.S.: Examining the Effects of Tutor Delivery Modes on Cognitive Presence and Learning Outcomes in Online Lectures. Journal of Educational Information and Media 13(4), 155-181 (2007) (in Korean)

11. Richardson, D.C., Spivey, M.J.: Eye-Tracking: Characteristics and Methods, 1-9. In: Wnek, G., Bowlin, G.: Encyclopedia of Biomaterials and Biomedical Engineering. Informa HealthCare (2004)

12. Poole, A., Ball, L.J.: Eye Tracking in Human-Computer Interaction and Usability Research: Current Status and Future Prospects. In: Ghaoui, C. (ed.) Encyclopedia of Human Computer Interaction, Idea group (2004)

13. Cowen, L.: An eye movement analysis of web-page usability. Masters by Research in the Design and Evaluation of Advanced Interactive Systems (2001)

14. Lohse, G.L.: Consumer Eye Movement Patterns on Yellow Pages Advertising. Journal of Advertising 26(1), 61-73 (1997)

15. Laarni, J., Isotalus, P., Kojo, I., Kärkkäinen, L.: Reading News from a Pocket Computer: An Eye-Movement Study. In: Harris, C.D., Duffy, V., Smith, M.J., Stephanidis, C. (eds.) Human-Centered Computing: Cognitive, Social and Ergonomic Aspects. The Proceedings of HCI International 2003, Lawrence Erlbaum, Mahwah (2003)

16. Cutrell, E., Guan, Z.: What Are You Looking for?: an Eye-tracking Study of Information Usage in Web Search. In: Proceedings of the SIGCHI conference on Human factors in computing systems, San Jose, California, USA (2007)

17. Jacob, R.J.K., Karn, K.S.: Eye Tracking in Human-computer Interaction and Usability Research: Ready to Deliver the Promises (Section commentary). In: Hyönä, J., Radach, R., Deubel, H. (eds.) The Mind's Eyes: Cognitive and Applied Aspects of Eye Movements, Elsevier Science, Oxford (2003)

18. Eyegaze Development System Information, http : / / www . eyegaze. com

19. Park, J., Lee, K.: Eyetrack - Developing Eyegaze Analysis Visualization Software for Designers' Use. In: KEER 2007, Sapporo, Japan, p. 10 (2007) 